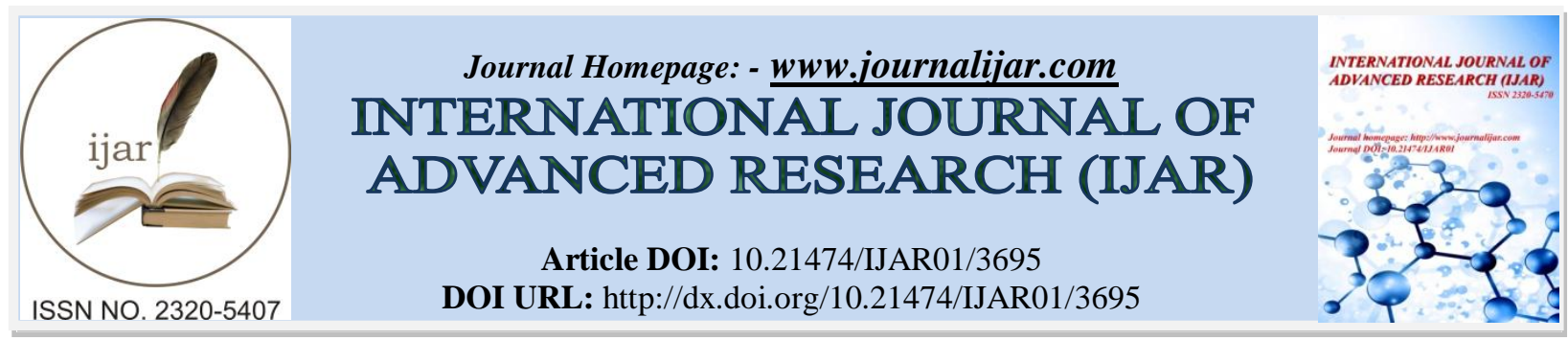

RESEARCH ARTICLE

\title{
TUMEUR GLOMIQUE DE L'HALLUX. NOUVEAU CAS ET REVUE DE LA LITTERATURE GLOMUS TUMOR OF HALLUX. NEW CASE AND REVIEW OF LITERATURE.
}

\author{
Omar Margad*, Jalal Boukhris, Mohammed Daoudi, Ouahb Azriouil, Aziz Mortaji and KH Koulali. \\ Service de traumatologie orthopédie de l'hôpital militaire Avicenne Marrakech.
}

\section{Manuscript Info}

Manuscript History

Received: 13 January 2017

Final Accepted: 10 February 2017

Published: March 2017

Key words:-

glomus tumor-hallux.

\section{Abstract}

- Glomus tumors are rare and benign, arising from a neuromyoarteriel glomus body. If the digital location is well known in surgery of the hand, the extradigitales locations suffer from a misunderstanding ending in diagnostic and therapeutic errors. We report a new case of tumor glomique of the hallux, and through a review of the literature, we would try to draw the attention towards these atypical location.

Copy Right, IJAR, 2017,. All rights reserved.

\section{Introduction:-}

Les tumeurs glomiques sont des tumeurs bénignes et rares qui se développent aux-dépends du glomus neuromyoartériel des anastomoses artério-veineuses.Elles touchent surtout les extrémités digitales, mais les localisations extra digitales ne sont pas rares mais surtout méconnues ce qui est responsable du retard de leur diagnostic et de leur prise en charge. Le but de notre travail est de rapporter un nouveau cas de tumeur glomique de l'hallux et d'attirer l'attention vers cette localisation inhabituelle.

\section{Cas Clinique:-}

Il s'agit d'une patiente âgée de 35 ans, sans antécédents pathologiques particuliers qui présente depuis 4 ans, des douleurs intermittentes siégeant à la base du gros orteil droit, coïncidant avec un traumatisme du même orteil. Nous avons reçu la patiente qui a consulté à plusieurs reprises vu l'augmentation de l'intensité des douleurs et l'absence d'amélioration sous traitement symptomatique. L'examen clinique de cette patiente montrait une décoloration de la base de l'ongle qui était très douloureuse à la palpation. L'examen ostéo articulaire de l'hallux droit était sans particularités .La radiographie standard ainsi que le bilan biologique étaient normales. L'IRM a objectivé un hyper signal autour du lit unguéal suggérant la possibilité d'une tumeur glomique [figure 1]. Une excision par voie latérale sous unguéale a été réalisée et l'examen anatomopathologique a confirmé le diagnostic de tumeur glomique .Les suites opératoires ont été simples, la patiente a eu un soulagement immédiat des douleurs et l'examen clinique de la patiente après un recul de 3 ans n'a pas montré de récidive de la symptomatologie.

\section{Discussion:-}

Les tumeurs glomiques sont rares et bénignes, elles représentent environ 1 à $5 \%$ de l'ensemble des tumeurs des tissus mous [1]. Ce sont des tumeurs qui touchent le sujet adulte, l'âge moyen est de 40 ans, elle est rare avant 20 ans [2]. Signalée pour la première fois par Wood en 1812 [3] qu'il a dénommé (nodule sous cutané douloureux), c'est Masson [4] en 1924 qu'il l'a définit en décrivant le glomus qu'il nomme ainsi à cause de sa similitude avec la glande vasculaire coccygienne de Luschka [5]. Si la localisation digitale est bien connue en chirurgie de la main [6], les localisations atypiques [7-13], comme notre cas au niveau de l'hallux, souffrent de méconnaissance, ce qui conduit à des erreurs diagnostiques et thérapeutiques. La symptomatologie clinique associe une triade classique 
avec douleurs sévères, point sensible et intolérance au froid. Ce qui caractérise également la symptomatologie est le contraste entre l'intensité des signes subjectifs et la pauvreté des signes objectifs. Rarement sont les cas où il ya une histoire traumatique comme dans notre cas [14]. Il n'y a pas d'imageries spécifiques permettant la confirmation diagnostique, cependant l'échographie, malgré sa faible spécificité aide à localiser la lésion [15].L'IRM reste le gold standard dans le diagnostic des tumeurs glomiques, elle précise le siège exact de la lésion et sa relation avec les structures avoisinantes [16-18].Son traitement est toujours chirurgical, il consiste en une exérèse chirurgicale qui entraine une disparition rapide des douleurs. Les récidives sont rares mais possibles, elles sont toujours précoces et le fait d'exérèse incomplète, d'où la recommandation de certains auteurs [18-20] d'exciser plus que les limites apparentes de la tumeur.

Figure 1:- aspect IRM objectivant un hyper signal autour du lit unguéal.

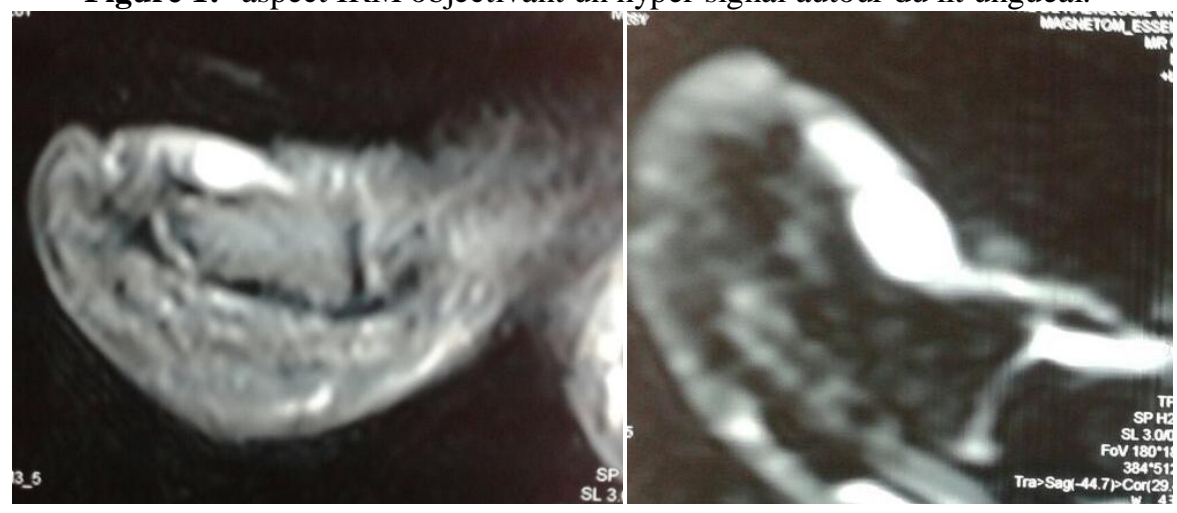

\section{Conclusion:-}

Les tumeurs glomiques sont rares mais non exceptionnelles. Elles peuvent siéger partout ou existe les glomi. Devant toute douleur avec ou sans masse palpable, sans étiologie évidente, le diagnostic de tumeur glomique doit être évoqué.

\section{References:-}

1. Akgun RC, Guler UO, OnayU. A glomus tumor anterior to the patellar tendon: a case report. Acta Orthop Traumatol Turc.2010; 44(3):250-253.

2. Johnson DL, Kuschner SH, Lance CS. Intraosseous glomus tumor of the phalanx: a case report.J Hand Surg Am.1993 Nov; 18(6):1026-8.

3. Wood W. On painful subcutaneous tubercle. Edinburgh Med J Surg.1812; 8:283-91.

4. Masson P. Le glomus neuromyo-artériel des regions tactile et ses tumeurs Lyon Chir 1924 ; 21 :257-280.

5. Glicenstein J, Ohana J, Leclercq C. Tumeurs de la main Berlin: Springer Verlag (1988).p.143-9

6. Carrol RE, Berman AT.Glomus tumors of the hand: review of the literature and report on twenty-eight cases. J Bone Joint Surg Am. 1972 Jun; 54(4):691-703.

7. A.Proietti, G.Ali, F. Quilici, P.Bertoglio, A.Mussi, and G.Fontanini.Glomus tumor of the shoulder: A case report and review of the literature.Oncol Lett. 2013 Oct; 6(4): 1021-1024.

8. Chun JS, Hong R, Kim JA. Extradigital glomus tumor: A case report.Mol Clin Oncol.2014 Mar; 2 (2):237-239.

9. Balaram AK, Hsu AR, Rapp TB, Mehta V, Bindra RR.Large solitary glomus tumor of the wrist involving the radial artery.Am J Orthop.2014 Dec;43(12):567-70.

10. Amillo S, Arriola FJ, Munoz G.Extradigital glomus tumor causing thigh pain:a case report.J Bone Joint Surg Br.1997 Jan;79(1):104-6.

11. Beksac K, Dogan L, Bozdogan N, Dilek G, Akgul GG, Ozaslan C.Extradigital glomus tumor of thigh.Case Rep Surg.2015;2015:638283.doi:10.1155/2015/638283.Epub 2015 Jul 8.

12. Gonçalves R, Lopes A, Julio C, Durao C, de Mello RA.Knee glomangioma:a rare location for a glomus tumor.Rare Tumors.2014 Dec 18;6(4):5588.doi:10.4081/rt.2014.5588.eCollection 2014.

13. Ayala H, Villas C, Canadell J.Glomus tumor.Acta Orthop Belg.1980 Mar-Apr; 46(2):219-25.

14. Koti M, Bhattacharryya R, Ewen SW, Maffulli N. Acta Orthop Belg. 2001Jun; 67(3): 297-9.

15. Smith KA, Mackinnon SE, Macauley RJ, Mailis A.Glomus tumor originating in the radial nerve:a case report.J Hand Surg Am.1992 Jul;17(4):665-7.

16. Dupuis P, Pigeau I, Ebelin M, Barbato B, Lemerle JP.The contribution of MRN in the study of glomus tumors.Ann Chir Main Memb Super.1994;13(5):358-62. 
17. Shih TT, Sun JS,Hou SM, Su TT.Magnetic resonance imaging of glomus tumor in the hand.Int Orthop.1996;20(6):342-5.

18. Matloub HS, Muoneke VN, Prevel CD, Sanger JR, Yousif NJ.Glomus tumor imaging:use of MRI for localization of occult lesions.J Hand Surg Am.1992 May;17(3):472-5.

19. Rettig AC, Strickland JW.J Hand Surg Am.1977 Jul; 2(4):261-5.

20. Varian JP, Cleak DK.Glomus tumors in the hand.Hand.1980 Oct; 12(3):293-9. 